\title{
Unanticipated late maturation of an arteriovenous fistula after creation of separate graft access
}

\author{
Hanzhou Li, Serena Jen, Tarun Ramayya, H. Gregory Bowers, Eran Rotem \\ Medical College of Georgia at Augusta University, Augusta, GA 30912, USA \\ Correspondence to: Hanzhou Li, BA. Medical College of Georgia at Augusta University, 1120 15th Street, Augusta, GA 30912, USA. \\ Email: hanli@augusta.edu.
}

Submitted Oct 10, 2017. Accepted for publication Jan 03, 2018.

doi: 10.21037/qims.2018.01.03

View this article at: http://dx.doi.org/10.21037/qims.2018.01.03

\section{Introduction}

Autogenous arteriovenous vascular access with an arteriovenous fistula (AVF) is the gold standard for attaining long-term hemodialysis vascular access (1). When compared to prosthetic arteriovenous grafts (AVGs) and tunneled hemodialysis catheters, an AVF has less frequent rates of thrombosis, stenosis, and infection. Additionally, AVFs are observed to provide a more long-term and stable access for hemodialysis patients $(2,3)$. A major obstacle to successful AVF creation is maturation, as about $30-50 \%$ of AVFs fail to mature. Maturation mostly occurs 4 to 6 weeks after the initial fistula surgery but that timeframe can vary with the average time ranging from 1 to 4 months. Thus, maturation of AVFs can be a relatively unpredictable process (4). Adequate AVF maturation is based on the National Kidney Foundation's Kidney Dialysis Outcomes Quantitative Initiative (KDOQI) guidelines which define a viable fistula as one that resides approximately $0.6 \mathrm{~cm}$ from the skin surface, has a flow $>600 \mathrm{~mL} / \mathrm{min}$, and has a diameter $>0.6 \mathrm{~cm}(5)$. AVFs that do not mature adequately within that time frame may be abandoned altogether and classified as early or primary failure $(6,7)$. Patients with primary AVF failure consequently rely more on grafts and catheters, exposing them to greater risks. Because maturation is a highly unpredictable and variable process, many AVFs that have not matured after several months are not followed up. Only a few cases have been witnessed to show delayed maturation of a fistula after abandonment after classification as primary failure and without any prior surgical intervention to salvage the immature fistula. However, no documentation currently exists to describe such findings.

\section{Case presentation}

A 69-year-old African American male with a history of hypertension (HTN), coronary artery disease (CAD), and end-stage renal disease (ESRD) on hemodialysis with a prior immature autogenous brachial-cephalic upper arm direct access fistula and a thrombosed prosthetic brachialbasilic loop access graft presented to interventional radiology (IR) for thrombolysis and angioplasty of prosthetic access in July of 2015. The patient underwent surgery for placement of autogenous brachial-cephalic upper arm access in June of 2014. Prior to surgery, preoperative vein mapping of the left upper arm with duplex ultrasound (DUS) showed a patent cephalic vein, a patent brachial vein, and a patent basilic vein that were 3,4 , and $4 \mathrm{~mm}$ in diameter, respectively. Autogenous brachialcephalic upper arm direct access was created in the left upper arm. Six weeks after surgery, cannulation from the autogenous upper arm access did not produce adequate flow, and DUS at the time showed that the autogenous brachial-cephalic access was smaller than $2 \mathrm{~mm}$ in size with flow less than $100 \mathrm{~mL} / \mathrm{min}$. After repeated failed attempts at cannulation from the fistula, patient was scheduled for prosthetic brachial-basilic loop access graft replacement in January of 2015. Vein mapping with DUS at this time showed immature autogenous brachial-cephalic upper arm access that was consistent with previous DUS with the brachial-cephalic upper arm access measuring smaller than $2 \mathrm{~mm}$ in size. A 7-mm prosthetic brachial-basilic loop access was created. After 3 months of successful hemodialysis with the prosthetic brachial-basilic loop access, poor flows and increased 


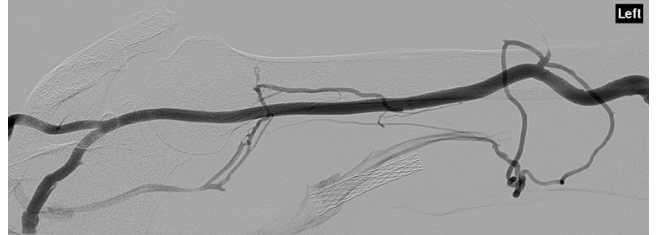

Figure 1 Venogram demonstrated a patent autogenous brachialcephalic upper arm access that had no intraluminal thrombus (7 $\mathrm{mm}$ in size).

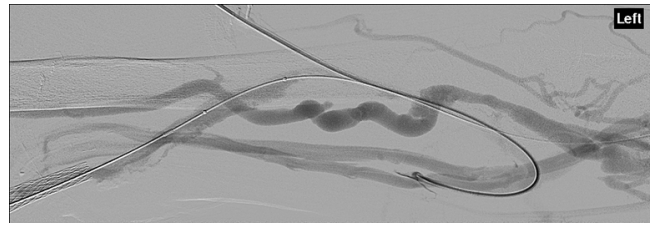

Figure 2 Venogram demonstrated high-grade stenosis throughout the outflow graft with intraluminal thrombus extending into the stent.

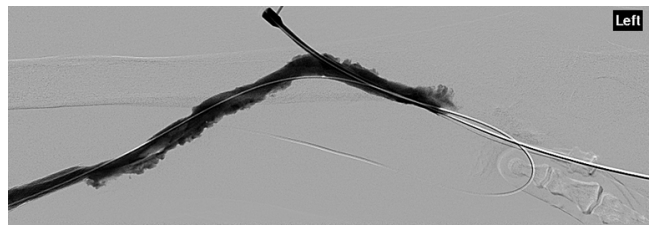

Figure 3 Final anterograde and retrograde venograms in the left arm demonstrated a patent prosthetic brachial-basilic loop access graft.

venous pressure were observed in the graft on DUS with flows measuring less than $200 \mathrm{~mL} / \mathrm{min}$. An auxiliary stent was placed on April 2015 to relieve the increased venous pressure. In June 2015, the patient presented to IR due to thrombosis of the prosthetic brachial-basilic loop access. Prior to the procedure, vein mapping with DUS showed thrombosed prosthetic brachial-basilic loop access and patent autogenous brachial-cephalic upper arm access that was $7 \mathrm{~mm}$ in size. Subsequently, anterograde access to the fistula was obtained by accessing the distal cephalic vein. Venogram demonstrated a patent autogenous brachial-cephalic upper arm access that had no intraluminal thrombus; it measured $7 \mathrm{~mm}$ in size (Figure 1). Anterograde access of the graft was obtained by accessing the distal basilic vein. Venogram demonstrated high-grade stenosis throughout the outflow graft with intraluminal thrombus extending into the stent (Figure 2). Venous angioplasty was performed with a $10-\mathrm{mm}$ balloon after administration of tissue plasminogen activator (tPA) at the central aspect of the prosthetic brachial-basilic loop access graft. Final anterograde and retrograde venograms in the left arm demonstrated a patent autogenous brachialcephalic upper arm access fistula along with a patent prosthetic brachial-basilic loop access graft (Figure 3). After the procedure, the patient had two vascular access sites in the left upper arm that were patent and adequate in size. Both the autogenous fistula and the prosthetic graft were left in place as viable access sites for hemodialysis. Upon discovering the patent autogenous fistula, the decision was made to proceed with thrombolysis and angioplasty of prosthetic graft, as there was no way of knowing whether the fistula would be adequate for hemodialysis. On subsequent hemodialysis visit, however, cannulation was successful from the original autogenous brachialcephalic upper arm access. DUS studies demonstrated the original autogenous brachial-cephalic upper arm access measuring $7 \mathrm{~mm}$ in size and flow measuring $700 \mathrm{~mL} / \mathrm{min}$. Initially, there was concern for arterial steal syndrome with presence of two access sites; however, the patient showed no evidence of pallor, necrosis, delayed pulses or any signs of claudication or weakness following the procedure.

\section{Discussion}

AVF maturation is a highly variable process and a major obstacle in obtaining long-term hemodialysis access. Early indications of fistula failure can clinically manifest as repetitive failed attempts at cannulation for dialysis and inadequate blood flow and size on DUS studies (7). If DUS studies show adequate flow and size during the initial evaluations of the AVF, the AVF is more likely to succeed. The autogenous brachial-cephalic upper arm fistula created in this patient had a high probability of failure due to various factors. This AVF had poor patency and flow on initial DUS studies, and the patient had several additional risk factors, such as CAD, ESRD, and African American ethnicity which further increases risk for primary failure (8). Even with the risk factors, the patient's AVF matured over a year after initial surgery. There are several possibilities as to why the fistula matured such a long time after surgery. The patient's genetics and comorbidities may have predisposed him to delayed maturation secondary to atherosclerosis and increased 
inflammation within the vessels $(5,9)$. Delayed maturation could also be the result of missed findings, such as preexisting venous stenosis or arterial stenosis, in the initial evaluations after the surgery. These kinds of lesions have been observed in $4 \%$ to $6 \%$ of patients with primary fistula failure $(9,10)$. Furthermore, a review by Rothuizen et al. describes several mechanisms specific to a patient with chronic kidney disease (CKD) which may inhibit fistula maturation. CKD can induce calcification and increased inflammation in the vascular system which impair a vessel's ability to distend upon high-flow stimulation that occurs in an AVF (10). Our case of delayed maturation illustrates the highly variable process of AVF maturation. Initially, the patient's immature fistula was not intervened on, and prosthetic graft eventually was placed, leading to a less effective and less safe method of access. This case provides an example of when failure to intervene early to salvage a fistula resulted in the placement of a prosthetic graft, which is associated with worse outcomes for patients. Examples such as these warrant the need to be more aggressive with fistula salvage, especially in patients with a high risk for primary failure.

\section{Acknowledgements}

None.

\section{Footnote}

Conflicts of Interest: This study was presented as poster at Cardiovascular and Interventional Radiology Society of Europe. Copenhagen, Denmark, October 15-20, 2017.

Informed Consent: Patients consent to the use of their anonymized images/information when they consent for any medical care/procedures done at the authors' academic institution thus we do not have direct written consent.

Cite this article as: Li H, Jen S, Ramayya T, Bowers HG, Rotem E. Unanticipated late maturation of an arteriovenous fistula after creation of separate graft access. Quant Imaging Med Surg 2018;8(4):444-446. doi: 10.21037/qims.2018.01.03

\section{References}

1. Brunori G, Ravani P, Mandolfo S, Imbasciati E, Malberti F, Cancarini G. Fistula maturation: doesn't time matter at all? Nephrol Dial Transplant 2005;20:684-7.

2. Allon $M$, Robbin ML. Increasing arteriovenous fistulas in hemodialysis patients: problems and solutions. Kidney Int 2002;62:1109-24.

3. Beathard GA, Arnold P, Jackson J, Litchfield T; Physician Operators Forum of RMS Lifeline. Aggressive treatment of early fistula failure. Kidney Int 2003;64:1487-94.

4. Asif A, Roy-Chaudhury P, Beathard GA. Early arteriovenous fistula failure: a logical proposal for when and how to intervene. Clin J Am Soc Nephrol 2006;1:332-9.

5. Patel ST, Hughes J, Mills JL Sr. Failure of arteriovenous fistula maturation: an unintended consequence of exceeding dialysis outcome quality Initiative guidelines for hemodialysis access. J Vasc Surg 2003;38:439-45; discussion 445.

6. Voormolen EH, Jahrome AK, Bartels LW, Moll FL, Mali WP, Blankestijn PJ. Nonmaturation of arm arteriovenous fistulas for hemodialysis access: A systematic review of risk factors and results of early treatment. J Vasc Surg 2009;49:1325-36.

7. Coentrão L, Turmel-Rodrigues L. Monitoring dialysis arteriovenous fistulae: it's in our hands. J Vasc Access 2013;14:209-15.

8. Hodges TC, Fillinger MF, Zwolak RM, Walsh DB, Bech F, Cronenwett JL. Longitudinal comparison of dialysis access methods: risk factors for failure. J Vasc Surg 1997;26:1009-19.

9. Dixon BS. Why don't fistulas mature? Kidney Int 2006; 70:1413-22.

10. Rothuizen TC, Wong C, Quax PH, van Zonneveld AJ, Rabelink TJ, Rotmans JI. Arteriovenous access failure: more than just intimal hyperplasia? Nephrol Dial Transplant 2013;28:1085-92. 